\title{
Consensus Report
}

\section{State-of-the-Art and New Options to Assess T Cell Activation by Skin Sensitizers: Cosmetics Europe Workshop}

\author{
Erwin van Vliet ${ }^{1}$, Jochen Kühnl ${ }^{2}$, Carsten Goebel ${ }^{3}$, Silvia Martinozzi-Teissier ${ }^{4}$, Nathalie Alépée ${ }^{4}$, \\ Takao Ashikaga ${ }^{5}$, Brunhilde Blömeke ${ }^{6}$, Aurélia Del Bufalo ${ }^{4}$, Magalie Cluzel $^{7}$, \\ Emanuela Corsini ${ }^{8}$, Nathalie Delrue ${ }^{9}$, Bertrand Desprez ${ }^{10}$, Nichola Gellatly ${ }^{11}$, Christoph Giese ${ }^{12}$, \\ Laura Gribaldo $^{13}$, Sebastian Hoffmann ${ }^{14}$, Martina Klaric ${ }^{10}$, Bernard Maillere ${ }^{15}$, \\ Dean Naisbitt ${ }^{16}$, Marc Pallardy ${ }^{17}$, Marc Vocanson ${ }^{18}$ and Dirk Petersohn ${ }^{19}$
}

${ }^{1}$ Services \& Consultation on Alternative Methods (SeCAM), Agno, Switzerland; ${ }^{2}$ Beiersdorf AG, Hamburg, Germany; ${ }^{3} \mathrm{COTY}$, Darmstadt, Germany; ${ }^{4}$ L'Oréal Research \& Innovation, Aulnay-sous-Bois, France; ${ }^{5}$ Shiseido Co., Ltd, Quality Assurance Center, Kanagawa, Japan; ${ }^{6}$ Department of Environmental Toxicology, University of Trier, Trier, Germany; ${ }^{7}$ LVMH Recherche, St Jean de Braye, France; ${ }^{8}$ Department of Environmental Science and Policy, Università degli Studi di Milano, Milan, Italy; ${ }^{9}$ Organisation for Economic Co-operation and Development (OECD), Paris, France; ${ }^{10}$ Cosmetics Europe, Brussels, Belgium; ${ }^{11}$ Unilever, Safety and Environmental Assurance Centre, Sharnbrook, Bedford, UK; ${ }^{12}$ ProBioGen AG, Berlin, Germany; ${ }^{13}$ Joint Research Centre, Institute for Health and Consumer Protection, Ispra, Italy; ${ }^{14}$ seh consulting + services, Paderborn, Germany; ${ }^{15}$ Commissariat à l'énergie atomique et aux énergies alternatives (CEA), Gif-sur-Yvette, France; ${ }^{16} \mathrm{MRC}$ Centre for Drug Safety Science, University of Liverpool, Liverpool, UK; ${ }^{17}$ Université Paris-Sud, Faculty of Pharmaceutical Sciences, Paris, France; ${ }^{18}$ French Institute of Health and Medical Research, Paris, France; ${ }^{19}$ Henkel AG \& Co. KGaA, Düsseldorf, Germany

\section{Summary}

Significant progress has been made in the development and validation of non-animal test methods for skin sensitization assessment. At present, three of the four key events of the adverse outcome pathway (AOP) are assessable by OECD-accepted in vitro methods. The fourth key event describes the immunological response in the draining lymph node where activated dendritic cells present major histocompatibility complex-bound chemically modified peptides to naive T cells, thereby priming the proliferation of antigen-specific T cells. Despite substantial efforts, modelling and assessing this adaptive immune response to sensitizers with in vitro T cell assays still represents a challenge. The Cosmetics Europe Skin Tolerance Task Force organized a workshop, bringing together academic researchers, method developers, industry representatives and regulatory stakeholders to review the scientific status of T cell-based assays, foster a mutual scientific understanding and conceive new options to assess $T$ cell activation. Participants agreed that current $T$ cell assays have come a long way in predicting immunogenicity, but that further investment and collaboration is required to simplify assays, optimize their sensitivity, better define human donor-to-donor variability and evaluate their value to predict sensitizer potency. Furthermore, the potential role of $\mathrm{T}$ cell assays in AOP-based testing strategies and subsequent safety assessment concepts for cosmetic ingredients was discussed. It was agreed that it is currently difficult to anticipate uses of T cell assay data for safety assessment and concluded that experience from case studies on real-life risk assessment scenarios is needed to further consider the usefulness of assessing the fourth AOP key event.

Keywords: skin sensitization, adverse outcome pathway, key events, T cell assays, safety assessment

Abbreviations: ACD, allergic contact dermatitis; AOP, adverse outcome pathway; BB, Bandrowski's base; BP, benzylpenicillin; CFSE, carboxyfluorescein succinimidy ester; DA, defined approach; DC, dendritic cell; DPRA, direct peptide reactivity assay; DNCB, 2,4-dinitrochlorobenzene; EC3, concentration inducing a 3-fold proliferation compared to concurrent vehicle-treated controls; ELISA, enzyme-linked immunosorbent assay; ELISpot, enzyme-linked ImmunoSpot; FACS, fluorescence-activated cell sorting; HLA, human leukocyte antigen; HSA, human serum albumin; hTCPA, human T cell proliferation assay; HuALN, human artificial lymph node model; IATA, integrated approach to testing and assessment; LLNA, local lymph node assay; LPS, lipopolysaccharide; IFN, interferon; IL, interleukin; KE, key event; MAPPs, MHCassociated peptide proteomics; MHC, major histocompatibility complex; MDDC, monocyte-derived dendritic cells; OECD, Organisation for Economic Co-operation and Development; PD, programmed death; PPD, para-phenylenediamine; PPRA, peroxidase peptide reactivity assay; PBMC, peripheral blood mononuclear cells; ST TF, Skin Tolerance Task Force; SMX-NO, nitroso-sulfamethoxazole; TNF, tumor necrosis factor; TD, toxicodynamic; TK, toxicokinetic; TNBS, trinitrobenzene sulfonic acid

Received September 1, 2017;

Accepted September 28, 2017;

Epub October 2, 2017;

doi:10.14573/altex.1709011

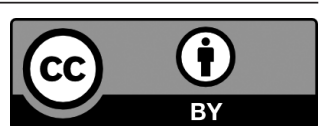

This is an Open Access article distributed under the terms of the Creative Commons Attribution 4.0 International license (http://creativecommons.org/ licenses/by/4.0/), which permits unrestricted use, distribution and reproduction in any medium, provided the original work is appropriately cited. 


\section{Introduction}

Skin sensitization is one of the major endpoints for the safety assessment of cosmetic ingredients but also other consumer products and industrial chemicals. Conventionally, the skin sensitization potential of cosmetic ingredients has been assessed using guinea pig models and more recently the murine local lymph node assay (LLNA), which measures the proliferation of lymphocytes in the draining lymph nodes of mice after application of a test substance on the dorsal surface of each ear once a day for three consecutive days (Kimber et al., 2002a; Gerberick et al., 2007). However, the $7^{\text {th }}$ amendment of the Cosmetic Directive implemented a prohibition on animal testing for cosmetic purposes (EC, 2009) irrespective of the availability of alternative, non-animal tests. Therefore, the area of skin sensitization has been at the center of efforts for the development, validation and acceptance of non-animal methods and testing strategies (Mehling et al., 2012; Reisinger et al., 2015; Ezendam et al., 2016).

Skin sensitization has been intensively studied and comprises two phases (Kimber et al., 2002b). The first phase (induction or sensitization phase) relates to the immunological events upon first exposure to a substance while the second (elicitation) phase describes the immunological response after a subsequent contact with the sensitizing substance, resulting in a type IV hypersensitivity reaction that may culminate in the clinical symptoms of allergic contact dermatitis (ACD). The adverse outcome pathway (AOP) for skin sensitization was recently described (OECD, 2012). The AOP outlines the sequence of chemical and biological processes required for the development of skin sensitization. The four key events (KEs) include: 1) the formation of hapten-protein conjugates by covalent binding of the sensitizing substance to skin proteins, 2) the activation of keratinocytes, which release pro-inflammatory signalling molecules, the subsequent 3 ) activation of dendritic cells (DCs), resulting in migration of DCs to the draining lymph node and subsequent presentation of hapten-protein conjugates to naïve $\mathrm{T}$ cells, and 4) the proliferation of antigen-specific effector and memory $\mathrm{T}$ cells.

Because of the complexity of the skin sensitization process, it is generally agreed that a single alternative test method is not sufficient to substitute animal models (e.g., LLNA) and that a combination of methods in integrated approaches to testing and assessment (IATA) will be required (Jowsey et al., 2006; Reisinger et al., 2015; Corsini et al., 2014; Ezendam et al., 2016; Jaworska, 2016). Several alternative, non-animal test methods have been developed that address the impact of a test substance on one or more AOP KEs (Reisinger et al., 2015; Wong et al., 2015; Ezendam et al., 2016). Currently, the first three of the four AOP KEs are assessable by OECD accepted methods, i.e., the Direct Peptide Reactivity Assay (DPRA) (OECD, 2015a; Gerberick et al., 2004), KeratinoSens (OECD, 2015b; Emter et al., 2010; Natsch et al., 2011), h-CLAT (Ashikaga et al., 2006; OECD, 2017), U-Sens ${ }^{\text {TM }}$ (Piroird et al., 2015; OECD, 2017) and the IL-8 Luc assay
(Kimura et al., 2015; OECD, 2017). In addition, several other promising assays addressing one or more KEs are either available or under development including VITOSens (Hooyberghs et al., 2008), peroxidase peptide reactivity assay (PPRA) (Gerberick et al., 2009), peripheral blood monocyte derived dendritic cell assay (PBMDC) (Reuter et al., 2011), Sensiderm (Thierse et al., 2011), SenCeeTox (McKim et al., 2012), Epidermal Equivalent IL-18 assay (Gibbs et al., 2013), GARD (Johansson et al., 2013), LuSens (Ramirez et al., 2014), and Sens-IS (Cottrez et al., 2015).

The integration of information derived from various of these assays for the skin sensitization AOP already provides information to predict the sensitizing properties of test substances, providing the basis for several defined approaches (DA) to be used in IATA (OECD, 2016). However, additional data on the biologically relevant induction of an antigen-specific $\mathrm{T}$ cell response may provide useful complementary information to complete the development of AOP-based DAs and IATAs and improve current predictive parameters.

The Cosmetics Europe Skin Tolerance Task Force (ST TF) is committed to providing regulatory accepted non-animal test strategies that would enable cosmetic industries to conduct skin sensitization safety assessments, primarily to allow marketing of new ingredients, but also to re-evaluate existing ingredients. Recognizing in this context the importance to consider the fourth AOP KE, the ST TF supported the development and refinement of a human $\mathrm{T}$ cell stimulation assay. The research advanced the understanding of how in vitro $\mathrm{T}$ cell information could be used for skin sensitization assessment and resulted in the development of the human $\mathrm{T}$ Cell Priming Assay (hTCPA) (Richter et al., 2013; Vocanson et al., 2014). However, the current assay protocol is still laborious and the results are difficult to interpret. Especially, analyzing the correlation between the number of proliferating $\mathrm{T}$ cell clones ( $\mathrm{T}$ cell receptor repertoire), as defined in the current in vitro setting, and the skin sensitizing potency of a substance, as defined by the EC3 value of the LLNA, did not reveal a clear contribution of the hTCPA for potency assessment.

For these reasons, the ST TF held a T cell workshop on June 27-28, 2016 in Brussels, Belgium to review the state-of-the art and accelerate the development of T cell-based assays to become informative for skin sensitization safety assessment. The main objective was to foster a mutual scientific understanding and discuss the opportunities to advance existing $\mathrm{T}$ cell assays with new options for read-out parameters, measurement technologies or more complex 3D cell models. During the workshop, scientists from academic institutions, test method developers, industry representatives and regulatory stakeholders presented their state-of-the-art science on $\mathrm{T}$ cell-based assays and discussed how the information provided by $\mathrm{T}$ cell assays could be used for the safety assessment of cosmetic ingredients. This report presents the main outcomes of the discussions and proposed recommendations from the invited experts during the workshop. 


\section{Current state-of-the-art T cell- based assays/approaches}

\subsection{The development and optimization of the human T Cell Priming Assay (hTCPA) for $T$ cell response assessment}

\section{Principles of the hTCPA}

The $\mathrm{T}$ cell response to haptenized proteins is antigen-specific and therefore the most unambiguous and informative endpoint for skin sensitization. The hTCPA has been developed to assess this hapten-specific $\mathrm{T}$ cell response induced by test substances (Dietz et al., 2010; Richter et al., 2013). The hTCPA utilizes immature monocyte-derived dendritic cells (MDDCs) and naïve $\mathrm{T}$ cells from healthy blood donors. To facilitate the maturation of the MDDCs, the cells are incubated with a non-cytotoxic concentration of the test substance and the toll-like receptor (TLR)4 agonist lipopolysaccharide (LPS). For T cell priming, the maturated test substance-pulsed MDDCs are co-cultured with isolated naïve $\mathrm{T}$ cells. After the priming stage, the $\mathrm{T}$ cells are re-stimulated with maturated MDDCs treated with the same substance (antigen specific re-stimulation), no substance (background control) or an irrelevant substance (specificity control). The induction of a test substance-specific $\mathrm{T}$ cell response is assessed by the detection of $\mathrm{T}$ cell proliferation, cytokine production, or the expression of cell surface markers (Richter et al., 2013; Vocanson et al., 2014).

The ST TF has closely followed and supported the development of the hTCPA. Multiple strategies have been implemented to enhance the sensitivity of the assay to detect test substance-specific $\mathrm{T}$ cell responses, including the depletion of several immune-regulatory cells (CD56+ and CD25+ cells) and use of CD1a+ MDDCs as potent antigen presenting cells (Vocanson et al., 2014). In 2012, a blinded study was performed on twelve reference sensitizers and four non-sensitizers using at least three donors. The readouts included $\mathrm{T}$ cell proliferation by thymidine incorporation and IFN $\gamma$ secretion analyzed by enzyme-linked immunosorbent assay (ELISA). The results for this limited set of compounds displayed a sensitivity of $67 \%$ and specificity of $100 \%$ (unpublished data). Due to a high inter-donor variability and several non-responding donors, it was recommended to test at least three to five donors per test substance, even for positive controls.

\section{ST TF supported project to refine and simplify the hTCPA}

In 2014, the ST TF supported a collaborative project between the University of Freiburg and INSERM in Lyon, France, to refine and simplify the hTCPA. The project aimed to 1) avoid the use of radioactivity by replacing thymidine incorporation with fluorescence-activated cell sorting (FACS) analysis of carboxyfluorescein succinimidyl ester (CFSE), IFN $\gamma$, Granzyme $\mathrm{B}$, IL-4, and TNF $\alpha, 2$ ) accelerate the conduction of the assay by generating mature dendritic cells in two instead of four days, so called "fast DCs", and 3) enhance T cell proliferation by blocking inhibitory checkpoint receptors such as programmed death (PD) 1 and cytotoxic T-lymphocyte-associated antigen
(CTLA) 4. The refined protocol was evaluated by testing three reference sensitizers and two non-sensitizers using five donors. The results showed that the refined assay was able to correctly predict the sensitizers and non-sensitizers. The most promising FACS read-out parameter was the expression of Granzyme B+ on CD8+ CFSE ${ }^{\text {low }} \mathrm{T}$ cells. Nevertheless, the new test protocol still showed limitations including a high inter-donor variability and background signals in the control cultures. Although FACS analysis could be used to track hapten-specific $\mathrm{T}$ cells, the method was found to be too cumbersome. Based on these findings, the project to further refine the hTCPA was continued. The fast-DCs were again replaced with standard four-day generated DCs to avoid the high background signals. Instead of FACS analysis, the enzyme-linked immunospot (ELISpot) method was used for IFN $\gamma$ and Granzyme B readouts to simplify the assay. To further improve the sensitivity, the number of primed $\mathrm{T}$ cells was amplified by increasing the rounds of co-culture with haptenized DCs.

The evaluation of the newly refined protocol using the same five test substances showed that the ELISpot method was far more convenient than FACS analysis. The number of IFN $\gamma$ producing cells was found to be the most predictive read-out parameter and the sensitivity and specificity of the assay for the five substances was comparable with previous evaluation studies. However, the ELISpot method required more T cells, restricting the number of testable substances to an inefficient level. A second round of culture for four days increased the number of primed $\mathrm{T}$ cells and thereby the sensitivity of the assay, but in some cases, it also increased the background level, probably due to autologous stimulation or response against serum albumin. Of note, a third round of stimulation did not induce a proliferation of T cells, but antigen-induced cell death. Moreover, the testing of three to five donors was deemed inevitable due to a remaining high donor-to-donor variability, including non-responding donors.

\section{Evaluation of the hTCPA and Alcyomics T cell assay by L'Oréal}

A study at L'Oréal evaluated the hTCPA for skin sensitization potential assessment by testing a strong (trinitrobenzene sulfonic acid (TNBS)), weak (eugenol) and non-sensitizer (lactic acid) using at least 5 donors. The read-outs that were used included the production of IFN $\gamma$ and TNF $\alpha$ measured by intracellular staining and/or by Luminex. Initial results showed that in the majority of cases the induction of IFN $\gamma$ and TNF $\alpha$ did not exceed background levels. The Luminex readout was more sensitive compared to intracellular staining. The Luminex IFN $\gamma$ and TNF $\alpha$ read-outs in combination with CFSE staining ( $\mathrm{T}$ cell proliferation) and CD69 expression ( $\mathrm{T}$ cell activation) were subsequently used to investigate the specificity of the test substance-induced $\mathrm{T}$ cell response during earlier stages of co-culture, before re-stimulation. The results showed that the maximum amount of proliferating T cells and CD69+ cells were induced by TNBS during day seven of culture. A follow-up study was performed testing the same three substances (TNBS, eugenol, lactic acid) at day 7 of culture measuring cytokines 
(IFN $\gamma$, IL-13, IL-5, TNF $\alpha$, RANTES, IP-10), CFSE and CD69. The results showed that the CFSE dilution was the most promising readout, but that the sensitivity was not sufficient to set up a skin sensitization assay.

In addition, to further investigate in the specificity of primary $\mathrm{T}$ cell response, L'Oréal evaluated a $\mathrm{T}$ cell assay developed by Alcyomics (Ahmed et al., 2016). The study tested three sensitizers (weak, moderate and strong) and two non-sensitizers using three doses and 10 donors. The CD14+ dendritic cells, derived from peripheral blood mononuclear cells (PBMC), and total $\mathrm{T}$ cells were co-cultured with the test substances for 7 days. The readouts included $\mathrm{T}$ cell proliferation using $\left[{ }^{3} \mathrm{H}\right]$-thymidine and IFNy secretion in the supernatants. The test results showed that all the sensitizers and non-sensitizers were correctly predicted for hazard and the two read-outs provided similar results, with surprisingly low inter-donor variability (unpublished data). Further investigation is required on more challenging compounds to confirm the encouraging results obtained.

\section{Remaining points for further refinement and optimization}

Taken together, substantial progress has been made to optimize and simplify the hTCPA to detect test substance-specific T cell responses. Nevertheless, further refinement and optimization is required to use the assay for the efficient and reliable assessment of skin sensitization potential. The main points that need to be addressed include:

- The hTCPA has shown a good specificity for the non-sensitizers that have been tested so far, however more work is required to improve the sensitivity of the assay to detect sensitizers. The dynamic range of the $\mathrm{T}$ cell responses in the hTCPA is not yet clear. It was discussed that a proliferative response of $25 \%$ of $\mathrm{T}$ cells in a heterologous setting depicts the maximum response. So far, the assay has been mostly evaluated with strong positive control sensitizers (2,4-dinitrochlorobenzene, trinitrobenzene sulfonic acid), showing a response of no more than $4 \%$ of $\mathrm{T}$ cells. The detection of moderate or weak sensitizers still needs to be further assessed.

- A major obstacle is the high inter-donor variability, which limits the reliability and reproducibility of the hTCPA test results. The difficulty is that not all blood donors show a substance-induced $\mathrm{T}$ cell response, which reflects reality, where, in an exposed population, only limited individuals become sensitized and develop ACD. This is in part linked to the human leukocyte antigen (HLA) system, the gene complex encoding the major histocompatibility complex (MHC) proteins, which are responsible for the regulation of the immune system in humans. The HLA haplotypes differ between human individuals. Together with environmental factors and individual immune system history, the HLA haplotype impacts the antigen presentation and stimulation of $\mathrm{T}$ cells in each donor. To overcome this donor-to-donor variability and the sometimes-low number of responders, it was concluded to test a high number of donors (at least five) to obtain a reliable prediction.
- It is not clear whether the assay has the capacity to predict the potency of skin sensitizers, which is a requirement for the performance of the safety assessment. Experiments aiming to examine the $\mathrm{T}$ cell receptor repertoire of proliferating $\mathrm{T}$ cells in the hTCPA did not show a clear correlation with the potency of skin sensitizers in vitro.

- There are concerns about the artificial in vitro micromilieu of the hTCPA, which limits the in vivo relevance of the assay. Unlike the in vivo situation, the induction of $\mathrm{T}$ cell responses in vitro requires the depletion of regulatory T cells by FACS and activation of autologous antigen presenting cells, e.g., using low-dose LPS. Additionally, multiple re-stimulations are required to assure the sufficiently high sensitivity of the assay. The disadvantage of these activation and re-stimulation steps is that they also increase the background level of the readouts.

- Despite the efforts to simplify the hTCPA, the current assay still depends on a laborious protocol and the long-term cultivation of a large number of cells from multiple human blood donors. This makes the assay time-consuming, costly and not suitable for screening purposes. In addition, it complicates transferability for future ring studies to evaluate accuracy and reproducibility. Therefore, the current hTCPA still needs further refinement and simplification.

\subsection{In vitro functional T cell assays for $T$ cell repertoire identification}

The presence of a $\mathrm{T}$ cell epitope is one of the key requirements for the induction of an antigen-specific $\mathrm{T}$ cell response. The $\mathrm{T}$ cell epitope for a substance of interest can be identified using in vitro functional $\mathrm{T}$ cell assays. For example, the INSERM UMR 996 laboratory at the University Paris-Sud in France developed a step-wise strategy to identify $\mathrm{T}$ cell epitopes using benzylpenicillin (BP) (Scornet et al., 2016).

First BP-human serum albumin (HSA) bioconjugates were synthesized using the intrinsic chemical reactivity of the nucleophilic distal amino group of lysine residues in HSA. This was followed by the identification of the BP-haptenated lysine residues using mass spectrometry analysis. In silico approaches were used to select the BP-hapten peptide conjugates with potential to act as epitopes for the induction of BP-specific CD4+ $T$ cells. The selected benzylpenicilloylated peptides (BP-Ps) were then synthesized and evaluated for their ability to activate naïve CD4+ T cells from healthy donors. DCs were loaded with the BP-HSA haptenized peptides and co-cultured with the autologous naïve CD4+ CD45 RA+ T cells. During co-culture the DCs primed the naive $\mathrm{T}$ cells, leading to the generation of HSA-BP-specific memory CD4+ T cells. The frequency of $\mathrm{T}$ cells responding to the generated BP-peptides was determined using ELISpot for IFN $\gamma$ and Poisson distribution. The test results revealed the ability of bioinspired peptides structurally stemming from BP-HSA to be recognized by naïve CD4+ T cells, thus identifying a pre-existing $\mathrm{T}$ cell repertoire for penicillin molecules bound to proteins. Some peptide sequences (epitopes) were favored and induced a higher frequency of responding HSA-BP-specific CD4+ T cells. 
Besides BP, the naïve $\mathrm{T}$ cell repertoire for nickel has been investigated. Until now only the response of $\mathrm{CD} 4+\mathrm{T}$ cells has been studied, since the generation of a CD8+ T cell response is more challenging due to the need for cross-presentation and potential cytotoxicity issues. It has been proposed that the size of the responding $\mathrm{T}$ cell repertoire may dictate, among other parameters, the strength of the immune response and potentially skin sensitization potency. However, there is no data available yet to support this hypothesis. The current approaches utilize HSA as a convenient reference protein for haptenization and peptide identification, however HSA may not be an ideal candidate (e.g., it only contains one accessible cysteine). The participants discussed that the complexity of the (modified) peptidome of cells is unlikely to be reflected under in vitro conditions. However, the choice of a reference protein or a well-defined mix of proteins could influence the chances to detect haptenization and the dynamic range of the $\mathrm{T}$ cell response. In addition, the microenvironmental conditions present during haptenization are likely to be a critical factor, potentially influencing the generation of different epitopes.

\subsection{T cell assay to predict immunogenicity of drug and cosmetic compounds}

Drug hypersensitivity is a serious adverse immunological reaction to an otherwise safe and effective therapeutic agent. The reactions tend to occur only in small subpopulations and are not related to the known pharmacology of a drug. Skin is the organ most commonly involved in drug hypersensitivity. Research into drug hypersensitivity involves the characterization of drug-specific $\mathrm{T}$ cell responses in the blood and/or skin of patients using a battery of in vitro assays in combination with LC-MS proteomics that address drug metabolism and the formation of drug protein adducts. A study on cutaneous hypersensitivity towards the antibiotic piperacillin (Sullivan et al., 2017) at the Centre for Drug Safety Science, University of Liverpool, studied a group of patients treated with piperacillin who developed a cutaneous hypersensitivity reaction. The study identified IL-22-secreting T cells from the blood and skin of patients as important mediators of this cutaneous hypersensitivity. Interestingly, skin-derived T cell clones were shown to be much more cytotoxic than blood-derived clones.

The center developed an in vitro $\mathrm{T}$ cell assay to assess the immunogenic potential of drugs (Faulkner et al., 2012). Naïve $\mathrm{CD} 4+\mathrm{T}$ cells and MDDCs were isolated from the peripheral blood of healthy volunteers. These cell types were co-cultured for eight days in the presence of the drug to expand the drug-responsive $\mathrm{T}$ cells. The expanded $\mathrm{T}$ cells were then incubated with new DCs and the drug to detect their antigen-specific response. The used readouts included proliferation (as measured using CFSE), cell phenotype (CD45RA/RO) and cytokine secretion (TNF, IL-5, IL-13) using ELISpot. To optimize the assay, the drug metabolite nitroso-sulfamethox- azole (SMX-NO) was used as a model drug allergen. T cells from all blood donors showed a dose-dependent dilution in CFSE (indicating increased proliferation), and the SMX-NOinduced $\mathrm{T}$ cell proliferation was associated with the secretion of cytokines (IFN $\gamma$, IL-5, IL-13). Moreover, the responding $\mathrm{CD} 4+\mathrm{T}$ cells changed from a naïve CD45RA+ phenotype to a CD45RO+ memory phenotype. Of note, the level of adducts detected in blood of patients was similar to the level required to activate $\mathrm{T}$ cells in vitro. Currently, the $\mathrm{T}$ cell assay has been used to characterize the $\mathrm{T}$ cell response to a variety of drugs and drug haptens.

The assay has also been used to study the $\mathrm{T}$ cell response to para-phenylenediamine (PPD) and Bandrowski's base (BB) using $T$ cells from healthy volunteers and hair dye allergic patients (Coulter et al., 2008; Gibson et al., 2015). The study results showed that $\mathrm{T}$ cells from allergic patients proliferated in response to both PPD and BB exposure. In contrast, $\mathrm{T}$ cells from non-allergic volunteers proliferated in response to $\mathrm{BB}$, but not to PPD exposure. These findings showed that the priming of naïve T cells to PPD failed, suggesting that an essential individual susceptibility factor may have been missing from the in vitro $\mathrm{T}$ cell priming assay or that PPD did not react to form a hapten.

The $\mathrm{T}$ cell assay has been simplified to develop an efficient one-step screening approach using naïve $\mathrm{T}$ cells to predict the allergenic potential of drugs. The assay uses $5-10 \mathrm{ml}$ of human blood to isolate the DCs and co-cultures them with frozen naïve $\mathrm{CD} 3+\mathrm{T}$ cells and the drug of interest. The use of frozen $T$ cells from a cell bank allows for multiple experiments using 40-50 donors each. The readout includes assessment of $\mathrm{T}$ cell proliferation by $\left[{ }^{3} \mathrm{H}\right]$-thymidine uptake and the assay provides information on both the strength and number of $\mathrm{T}$ cell responses against the drug compound. In combination with the establishment of an HLA-typed PBMC bank (including samples from 1000 healthy volunteers), the assay will be used to screen HLA-restricted drug hypersensitivity reactions.

\subsection{New in silico and in vitro options to assess $\mathrm{T}$ cell activation}

\section{In silico methods to predict immunogenicity}

The available in silico methods aim to predict the interactions of specific peptides with HLA molecules. The principles of the methods include peptide alignments, scoring matrices, structural analysis and learning algorithms. The advantages of in silico methods are that they are easy to use, not expensive and available via web resources, e.g., the Immune Epitope Database $^{1}$. In silico methods are commonly used in early steps of drug development for preliminary immunogenicity assessment and $\mathrm{T}$ cell epitope mapping. The available methods have shown very good allele-dependent predictions of peptide binders. Moreover, they have been able to predict $\mathrm{CD} 4+\mathrm{T}$ cell epitopes (Wang et al., 2008).

1 http://www.immuneepitope.org 
HLA class II binding assays

HLA class II binding assays can be used to evaluate the binding affinity of compounds for multiple HLA class II molecules. The assays are high-throughput and based on either competitive ELISA/radioimmunoassay or direct assays using purified HLA Class II molecules. The experimental data provides the binding frequency for multiple HLA alleles. The assays are limited to preponderant alleles and were found to be overpredictive. Nevertheless, many $\mathrm{T}$ cell epitopes have been identified for therapeutic proteins, allergens, viruses and tumor antigens (Texier et al., 2000, 2001; Castelli et al., 2002).

\section{Peptide elution and mass spectrometry sequencing methods}

Peptide elution and mass spectrometry sequencing methods have the objective to identify MHC Class II bound peptides processed and presented by dendritic cells (DCs). The methods are based on MHC associated peptide proteomics (MAPPs) assays. This approach allows investigating the effect of protein aggregation and formulation on peptide presentation by DCs (Rombach-Riegraf et al., 2014).

\section{T cell amplification assay}

The $\mathrm{T}$ cell amplification assay allows quantification of the size of the naïve CD4+ $\mathrm{T}$ cell repertoire. The assay has been used for the quantitative analysis of the $\mathrm{CD} 4+\mathrm{T}$ cell repertoire specific to therapeutic proteins and vaccine candidates (Delluc et al., 2011; Castelli et al., 2013). The assay uses PBMCs from healthy donors with different HLA-DR related allotypes to generate antigen-specific CD4+ $\mathrm{T}$ cell lines. The CD4+ $\mathrm{T}$ cells are isolated using anti-CD4 monoclonal antibodies coupled to magnetic microbeads. The isolated $\mathrm{CD} 4+\mathrm{T}$ cells are then primed by co-culture with autologous DCs loaded with the therapeutic protein or antibody of interest. The CD4+ $\mathrm{T}$ cells are re-stimulated on days 7 and 14 with fresh autologous DCs loaded with the protein or antibody to induce further $\mathrm{T}$ cell proliferation. The specificity of the $\mathrm{CD} 4+\mathrm{T}$ cell lines was investigated using ELISpot for IFN $\gamma$ on day 21 of culture. The results demonstrated the ability of the assay to anticipate the immunogenicity of the therapeutic protein based on the quantification of the number of specific T cells per millions of cells. By testing multiple proteins/antibodies or reference compounds, the relative immunogenicity could be ranked. Of note, characterization of the affinity of peptides to HLA-DR did not display a clear correlation between good binding properties of the peptide and its ability to act as a strong stimulator of $\mathrm{T}$ cell responses. Hence, it was argued to characterize the potential interaction of a peptide with HLA molecules as a pre-requirement for $\mathrm{T}$ cell stimulation, but not to use its HLA-binding affinity to predict its $\mathrm{T}$ cell stimulation properties. Limitations of the assay include the required long-term 21-day cell culture period and high dependence on the number of $\mathrm{T}$ cells added to each well of the cell culture plate.

\subsection{The human artificial lymph node model (HuALN)}

ProBioGen AG have developed a human artificial lymph node model (HuALN), which can be used to assess the immune stimulation, immunogenicity or immunotoxicity of pharmaceutical or chemical substances (Giese and Marx, 2014). This bioreactor model mimics the in vivo conditions of the human lymph node by containing PBMCs of healthy donors in a continuously perfused 3D hydrogel containing arginine-glycine-aspartate (RGD) sequences. The HIRISTM bioreactor device currently used for the HuALN model is able to incorporate a large cell repertoire $\left(10^{8}\right.$ cells $)$ for a long-term culture of up to 28 days. This set up allows the analysis of the effects of substances or pharmaceutics on immune cells, including repeated dosing or antigen re-stimulations. To this end, the cells in the bioreactor can be re-stimulated with pre-exposed DCs plus antigen (the latter to stimulate PBMCs). The model is suitable for inline or endpoint analysis using in situ imaging or the analysis of cells or culture media supernatants. The model has been used to assess the immune stimulation or immunogenicity of drug compounds by analyzing the cells' cytokine phenotype or antibody response from different donors during the 28 days of culture. In addition, the model has been used for mechanistic immunological studies, i.e., the activation of T helper (Th) 1 and Th2 pathways. The current developments include the implementation of stromal cells (MSCs) to improve tissue organoid formation and designing a miniaturized assay format. Benchmark substances are currently evaluated to characterize and standardize the rate of response. Limitations of the model include restrictions due to donor-to-donor variations and background responses. In particular, weakly immunogenic proteins are difficult to assess. A miniaturized bioreactor device ( $\mu \mathrm{ALN})$ using a microfluidic chip format is under development to facilitate multi-donor studies in parallel.

\subsection{Toxicokinetic/toxicodynamic (TK/TD) skin allergy assessment (SARA) model}

Unilever have developed a toxicokinetic and toxicodynamic skin allergy assessment model that aims to mathematically model the induction of skin sensitization to enable the prediction of a safe level of skin exposure (MacKay et al., 2013; Maxwell et al., 2014). The mechanistic model is based on the assumptions that 1) the extent of naïve $\mathrm{CD} 8+\mathrm{T}$ cell receptor triggering is a key determinant of allergic status, 2) there exists at least one $\mathrm{T}$ cell specific to the antigen, 3 ) the required $\mathrm{T}$ cell co-stimulatory signals are sufficient, 4 ) the CD4+ T cell response is optimal and 5) DC migration from exposure site is adequate. The benchmark for the model was the prediction of human data for the direct-acting sensitizer 2,4-dinitrochlorobenzene (DNCB).

The TK model describes DNCB kinetics in skin due to its diffusion and partitioning into the viable layers of skin, clearance by dermal capillaries and the covalent modification of protein nucleophiles. The kinetic parameter values used in the model were obtained from scientific literature or determined 
by in vitro experiments using radiolabeled DNCB. The TD model describes the biological response due to proteasome processing of protein nucleophiles to form small peptides, transport to the endoplasmic reticulum, the binding of peptides and hapten-peptide complexes to MHC-I molecules and transport to plasma membrane, the binding of peptide-MHC and hapten-peptide-MHC to $\mathrm{CD} 8+\mathrm{T}$ cell receptors, the subsequent activation and expansion of naïve antigen-specific CD8+ T cells. For the TD model, the AOP was used to establish the mechanisms and the parameter values were obtained from literature data. Sensitivity analysis was performed on the TK and TD models to assess which parameters had the largest effects on the predictions.

The model was parameterized to simulate the exposure scenario of Friedmann et al. (1983). The results showed that the TK/TD approach provides acceptable predictions of the sensitizing dose of DNCB in humans under this exposure scenario. Further work is required in order to determine whether this approach has predictive capacity for other known sensitizers and how further in vitro data can be incorporated to predict dose thresholds for human sensitization.

\section{Recommendations and remaining questions on T cell-based assays to evaluate skin sensitization}

\subsection{Improving the sensitivity of $T$ cell assays}

The use of a second round of re-stimulation was shown to increase the sensitivity of the hTCPA. The optimal number of re-stimulations seems to be limited to two, as T cells did not respond to a third round of re-stimulation. To further improve the sensitivity of $\mathrm{T}$ cell assays it was recommended to investi- gate the addition of supplementary cytokines (i.e., IL-2) during the re-stimulation steps. The additional cytokines may also be able to reduce the background responses in the ELISpot assay. Another approach that might increase the sensitivity of the assay would be the coupling of test substances to proteins to form hapten-protein conjugates. Though HSA is a convenient candidate to use for lysine binders, alternative proteins, e.g., from skin cells, should be considered. For example, the coupling of test substances to proteins and exposure of DCs to the haptenated proteins would facilitate a more specific response to test substances and address more specifically the response to immunogenic peptides. The disadvantage of this approach is that it increases the complexity of the assays and needs careful characterization of the conjugates (e.g., using mass-spectrometry).

\subsection{Inter-donor variability and reproducibility}

A major challenge for T cell-based assays is the wide response spectrum in the $\mathrm{T}$ cell responses to a skin sensitizer between different human blood donors, ranging from non-responding to highly responding donors. This inter-donor variability certainly reflects in part the diversity of responses observed in human populations exposed to a skin sensitizer, where only some individuals become sensitized and develop ACD. It is not clear what determines whether an individual will or will not become allergic after exposure to a skin sensitizer. Multiple parameters (e.g., sex, age, ethnicity, HLA haplotype) have been studied, but only very few associations or explanations have been described (Emtestam et al., 1993; Burel et al., 2017). It has been hypothesized that this may be linked to the $\mathrm{T}$ cell precursors available in the $\mathrm{T}$ cell repertoire of an individual blood donor. However, the exposure conditions play a crucial role as well,

Tab. 1: Reference substances for the development and evaluation of T cell-based assays

\begin{tabular}{|l|l|l|l|l|}
\hline Substance name & CAS\# & $\begin{array}{l}\text { Human Potency } \\
\text { Category }\end{array}$ & LLNA Potency Category & Description \\
\hline 2,4-Dinitrochlorobenzene (DNCB) & $97-00-7$ & 1 & Extreme & Pos. control \\
\hline Trinitrobenzene sulfonic acid (TNBS) & $2508-19-2$ & - & Strong & Pos. control \\
\hline $1,4-$ Phenylenediamine & $106-50-3$ & 1 & Strong & \\
\hline Glyoxal & $107-22-2$ & 2 & Moderate & Moderate \\
\hline Resorcinol & $108-46-3$ & 4 & Weak & \\
\hline Eugenol & $97-53-0$ & 3 & Weak & Neg. control \\
\hline Cinnamic alcohol & $104-54-1$ & 3 & Non-sensitizer & Neg. control \\
\hline Lactic acid & $50-21-5$ & 6 & Non-sensitizer & Neg. control \\
\hline Salicylic acid & $69-72-7$ & 6 & Non-sensitizer & Specificity control \\
\hline Glycerol & $56-81-5$ & 6 & Moderate & \\
\hline Sodium lauryl sulfate & $151-21-3$ & 6 & 1 & \\
\hline
\end{tabular}

1 Human potency category from Basketter et al. (2014).

2 Modified mean LLNA EC3 value from Hoffmann et al. (2018). 
as it is generally agreed among the scientific community that if the dose of a strong or extreme sensitizer is high enough, nearly all donors will be sensitized (Friedman et al., 1983), while exposure to a weak or moderate sensitizer will only sensitize a select number of donors with a larger range of responses.

To deal with the wide $T$ cell response spectrum, it was proposed that the inter-donor variability could be mapped in a database by testing the $\mathrm{T}$ cell response magnitude for a large number (> 50) of blood donors reflecting the most commonly represented HLA haplotypes of the human population. This information may then be used to characterize a hapten-specific $\mathrm{T}$ cell response frequency in a human population and could be integrated to optimize the subsequent experimental set-up of $\mathrm{T}$ cell-based assays to reduce the inter-donor variability by pre-selection of donors. Besides the inter-donor variability, it is also important to determine the intra-donor variability in the $\mathrm{T}$ cell-based test system to quantify reproducibility of repeat experiments using the same donor over time.

\subsection{Skin sensitizer potency prediction}

Until now, no clear correlations have been observed between the potency of sensitizing substances and relative $\mathrm{T}$ cell proliferation in $\mathrm{T}$ cell-based assays. It has been suggested that more $\mathrm{T}$ cell clones can respond to a strong sensitizer compared to a weak sensitizer (Kimber et al., 2012; Esser et al., 2014), however to date no data has been published to support this hypothesis. Further studies are required to understand how sensitizer potency and the $\mathrm{T}$ cell response are related. To obtain a first indication, the potential correlation between the number of IFN $\gamma$ producing cells in the hTCPA and the potency or concentration of a test substance could be investigated using a set of reference substances. Table 1 provides a list of reference substances for the development and evaluation of $\mathrm{T}$ cell-based assays proposed by the workshop participants.

In addition, the relationship between sensitizer potency and the $\mathrm{T}$ cell repertoire remains to be clarified. As previously mentioned, it is hypothesized that the size of the naïve $\mathrm{T}$ cell repertoire relates to immunogenicity and may also dictate the strength of the T cell response (Kimber et al., 2012; Esser et al., 2014). Therefore, also the size of the responding $T$ cell repertoire should be investigated after exposure to reference substances with different potencies. Measurement of the T cell repertoire is, however, challenging and was shown to vary with the technologies used (Hou et al., 2016).

\subsection{Link between haptenization and immunogenicity}

Another knowledge gap is the question whether and how the formation of hapten-protein conjugates determines the potency of a sensitizer. It would be expected that generation of a large amount of haptenized protein would increase the probability that peptides from these proteins are presented in MHC molecules, thereby increasing the probability of a $\mathrm{T}$ cell response. A possible experiment to confirm this hypothesis would be to identify haptenated peptides present in the pocket of MHC class II and I molecules using a MAPPs assay (elution of peptides from $\mathrm{MHC}$ molecules followed by identification using mass-spectrometry) and to then correlate the quantity of haptenated peptides present in the HLA pocket with the $\mathrm{T}$ cell response. However, information on the $\mathrm{T}$ cell recognition of these peptides is still missing and a few immunodominant peptides could already give a strong response.

In addition, it was suggested to combine model peptides and test substances in different concentrations to investigate whether haptenization correlates with the strength of the immune response, e.g., by using an in chemico assay such as the DPRA (Gerberick et al., 2004). A key challenge for this type of study will be to emulate the in vivo environment in which haptenization takes place. The DPRA uses a synthetic peptide, which may bind differently to MHC and influence the results. In addition, there is no clear understanding what proteins/peptides would be most relevant to test. Since the affinity/avidity of DC $\mathrm{T}$ cell interaction is key, another significant difficulty is that the responses to test substances combined with a limited number of model peptides will not be representative of the complexity of the possible $\mathrm{T}$ cell hapten-peptide interactions.

\subsection{Applicability domain}

So far, only a limited number of substances have been tested in $\mathrm{T}$ cell based-assays. To understand the applicability domain of $\mathrm{T}$ cell assays, evaluation of more substances covering a wide chemical space is essential. One of the key limiting factors for in vitro assays, including those based on $\mathrm{T}$ cells, is the solubility of the test substances in the cell culture medium. It has been shown that the response of DCs to (hydrophobic) allergens is increased if hydrophobic test substances are encapsulated with nanoparticle vectors (Cortial et al., 2015). The encapsulation helps to overcome the solubility limitations and boosts the capture of the hapten and subsequent $\mathrm{T}$ cell response. However, although the nanoparticle encapsulation of DNCB significantly increased the ability of DCs to prompt specific $\mathrm{T}$ cell activation and thus assay sensitivity (Cortial et al., 2015), the additional step made the assay more complex.

\subsection{Importance of human leukocyte antigen system}

The role of the HLA system in skin sensitization is currently unclear. The current assumption is that for stronger allergens the $\mathrm{T}$ cell response is less dependent on HLA haplotype compared to that for weak allergens. To better understand the role of the HLA response, a screening study would be required, comparing hundreds of human blood donors for a substance of interest. Up to now, about 30 HLA types have been identified as playing a role in $\mathrm{T}$ cell responses to substances (White et al., 2015), but thousands could be involved. For the development of a general $\mathrm{T}$ cell assay to assess skin sensitization potential, dominant HLA alleles could be introduced in the panel to be as close as possible of the diversity of the population. However, response to substances with a low potency might be missed if the implicated alleles are at low frequency in the population and may require repeat testing for different geographies as different haplotypes dominate in different regions. 
Of note, it should be mentioned that the LLNA, which has been used as a reference standard to assess skin sensitizing potential in the past, is based on a very limited set of MHC molecules (as inbred mouse strains are used), which in turn differ from the human HLA molecules. In contrast, the results of human repeat insult patch test (HRIPT) studies are more relevant, since they represent a wider survey of human MHC variants.

\section{The importance of $T$ cell-based assays for the development of integrated testing approaches}

It is commonly agreed that the assessment of skin sensitization potential without animal testing requires a combination of alternative methods in integrated approaches (Jowsey et al., 2006; Reisinger et al., 2015; Ezendam et al., 2016; Jaworska, 2016). The AOP plays an important role in the development of integrated approaches because it provides a scientific framework to cover the mechanistic steps and KEs of the skin sensitization process. Several DAs have been submitted to the OECD, each integrating data from non-animal test methods that address one or more AOP KEs (OECD, 2016). The Cosmetics Europe Skin Tolerance Task Force and the National Toxicology Program Interagency Center for Evaluation of Alternative Toxicological Methods (NICEATM) have evaluated several of these DAs using a comprehensive database with non-animal test method, LLNA and human skin sensitization data (Hoffmann et al., 2018; Kleinstreuer et al., 2018).

The question of how to integrate a $\mathrm{T}$ cell assay result into a safety assessment strategy was extensively discussed. From a mechanistic point of view, the currently developed AOP-driven IATAs do not cover all four AOP KEs because they are based on the assessment of innate immune responses within the first three KEs. The fourth KE, involving activation of the adaptive immune system, has not yet been integrated, as the required $\mathrm{T}$ cell-based assays are not yet available. However, it remains to be seen if and under which circumstances a test method addressing KE four can be of added value. Because the induction of a $T$ cell response is one of the requirements for skin sensitization, the assessment of the first three KEs provides valuable, but not definite scientific evidence for skin sensitization hazard prediction. Nevertheless, when combined in an IATA, the test results of the first three AOP KE assays may be able to provide sufficient information for hazard identification (e.g., substances positive for the first three KEs). Consequently, if it is not possible to generate potency data using $\mathrm{T}$ cell assays, an additional positive $\mathrm{T}$ cell result would only confirm the initial hazard identification decision and not the subsequent risk assessment steps in a safety assessment strategy. Since the T cell-based assays still need to undergo substantial further development, it is not foreseeable when they will reach a level of standardization that would enable them to add value to skin sensitization safety assessment. Once at that stage, it is likely that they will not be suitable for routine testing of many substances due to their low-throughput nature, but potential use scenarios, e.g., supporting the safety assessment of novel substances and challenging weak and non-sensitizing substances with equivocal test data from other assays, are conceivable.

A recent publication by Vukmanović and Sadrieh (2017) described additional mechanisms for the induction of skin sensitization, which are independent from the current AOP, involving the presentation of hapten-carrier peptides to $\mathrm{T}$ cells. The existence of these suggested mechanisms (incl. pharmacological interactions with immune receptors, HLA groove blockade, peptide exchange and cryptic epitope presentation) could explain the imperfect concordance between in vitro assay data based on the hapten/carrier concept and animal/human reference data. The authors pointed out the importance of developing and applying $\mathrm{T}$ cell-based assays, as they are the only downstream test methods able to detect immunogenic potential resulting from all possible mechanisms of skin sensitization, including those not yet covered by the current AOP.

\section{The anticipated use of $T$ cell-based assays for AOP-based safety assessment}

Safety assessment integrates different types of information on exposure, physicochemical properties and testing results to define a safe human dose for a test substance. In Europe, the implementation of the $7^{\text {th }}$ Amendment of the Cosmetic Directive (EC, 2009) imposed a complete ban on animal testing of ingredients with a cosmetic purpose. Hence, no LLNA potency information can be generated for substances using the LLNA and, consequently, information from alternative non-animal test methods has to be used instead. The transition from in vivo to in vitro information is a challenging process, as confidence needs to be acquired in the testing approaches, as well as a new understanding of uncertainty factors. With the documentation of the skin sensitization AOP, safety assessment has become more mechanistic pathway-driven by the curation of information for the different mechanisms involved. A key question remains as to which and how many AOP KEs need to be covered to enable a robust safety assessment for a given substance.

The antigen-specific $\mathrm{T}$ cell response is an essential part of the skin sensitization process and although it may not be a prerequisite, information on this fourth KE could provide complementary scientific evidence to support a safety assessment approach. If a given test substance is unable to induce a $\mathrm{T}$ cell response, it may be possible to conclude that it does not cause skin sensitization, provided the $\mathrm{T}$ cell assay is sufficiently sensitive. For a pragmatic safety assessment, it needs to be considered what additional reassurance, if any, is required if data covering the first three KEs indicate a sensitizing potential. A key open question for the use of T cell-based assays in safety assessment is how many responding donors would be required to obtain sufficient confidence in the test result.

Correspondingly, $\mathrm{T}$ cell assays may provide valuable information to support the safety assessment of chemical sensitizers depending on the data available for the individual safety as- 


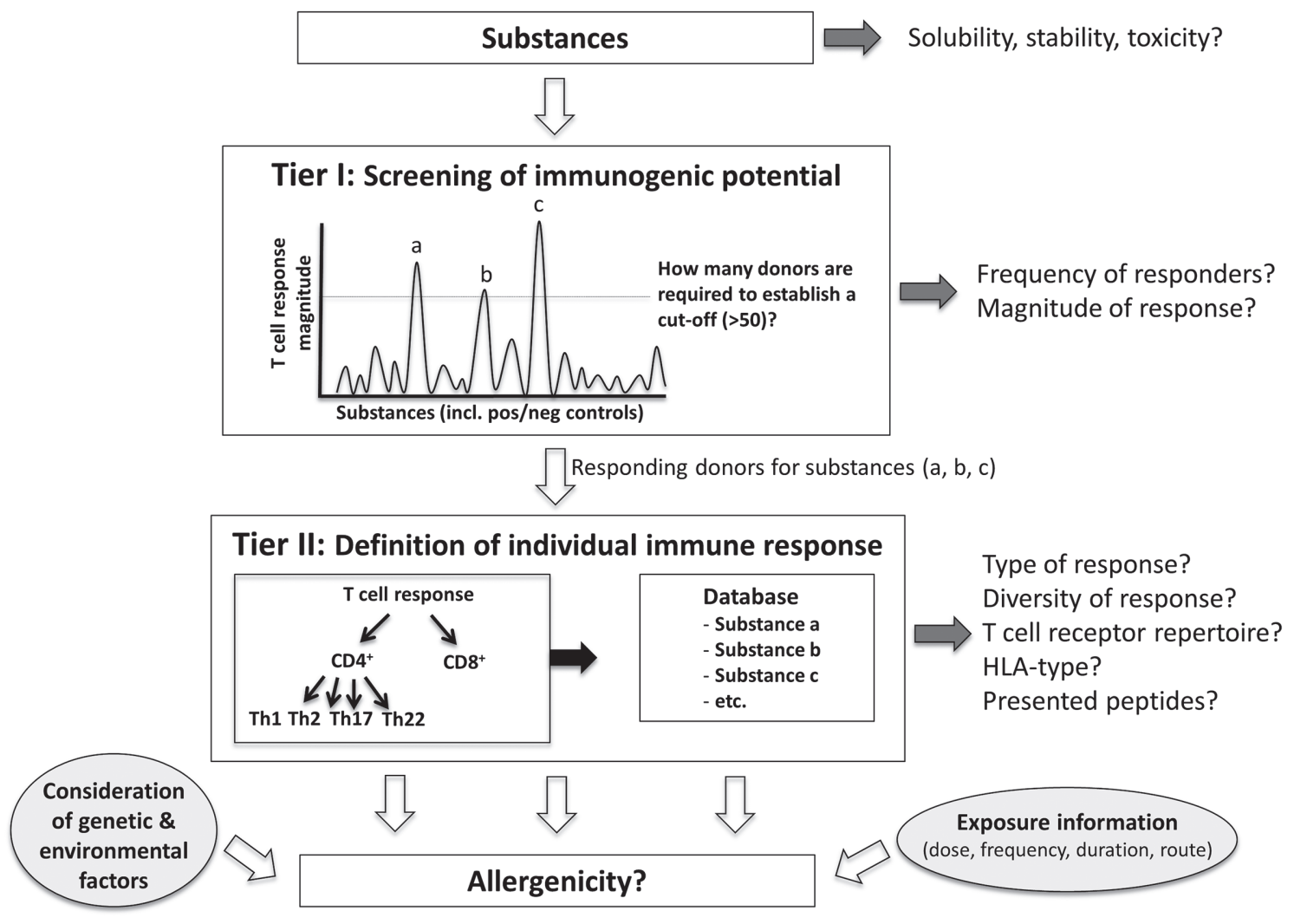

\section{Fig. 1: T cell assay-based tiered testing approach for immunogenicity and allergenicity assessment}

T cell-based assays may provide valuable information on the immunogenic potential and allergenicity of substances. To improve efficiency, this information would likely be generated using a tiered approach. In Tier I, the screening of immunogenic potential using in vitro $\mathrm{T}$ cell responses based on blood samples from human donors may generate information on the ratio of responding donors towards a given substance and the variability of T cell response magnitude. In Tier II, more complex T cell-based assays could provide more detailed mechanistic information on the antigen-specific $T$ cell response at multiple concentrations using cells from responding donors in Tier I. The assays could evaluate different parameters including the T cell receptor repertoire, HLA type, peptide presented by DCs and T cell subset activation. By testing substances with different properties in multiple human donors, a database could be established that covers a wide range of substances. The immune response data in combination with exposure information and considerations of genetic and environmental factors could be used to assess the allergenicity of a given substance.

sessment. To improve testing efficiency, this information would likely be generated using a tiered approach (See Fig. 1).

- Tier I: The assessment of immunogenic potential of a given substance based on in vitro $\mathrm{T}$ cell responses based on blood samples from human donors $(>50)$ may generate information on the frequency of responding donors towards a given substance and the variability of $\mathrm{T}$ cell response magnitude.
Using this information, we may be able to deduce the risk that an individual will develop an adaptive immune response after exposure to the substance. The frequency of responding donors could be considered to provide information on the substance's relative strength and thus be useful for the safety assessment approach. It is expected that a strong sensitizer is able to sensitize a high ratio of people and therefore 
would induce a $\mathrm{T}$ cell response in a great number of blood donors.

- During Tier II, more complex T cell-based assays could provide detailed mechanistic information on the induction of an antigen-specific $\mathrm{T}$ cell response at multiple concentrations of a test substance using cells from responder donors (from Tier I). The assays could evaluate different parameters including the magnitude of the proliferative response, the T cell receptor repertoire, the T cell subsets activated (Th1, Th2, Th17, etc.), and whether the response is $\mathrm{CD} 8+$ or $\mathrm{CD} 4+\mathrm{T}$ cell mediated. For the magnitude of the proliferative response different endpoints will have to be used, i.e., the number of responding cells using ELISpot and the level of cell activation by cytokine production or proliferation measurements. The EC50 (effective concentration that gives $50 \%$ of the maximum response) could be used as a parameter. By testing substances with different properties and potencies, a database could be established that covers a large applicability domain (e.g., cosmetic ingredients). This tier will provide information on the in vitro concentration at which a substance will induce an immune response, the type of response and the strength of the response via comparison with benchmark substances. Such information could be used to define a safe exposure level. This implies that an agreed way to translate results from the in vitro assays (e.g., effective concentration or response frequency) to a metric value that can be used to define a safe human dose would be available.

To learn how the information from $\mathrm{T}$ cell-based assays could support safety assessment it was recommended to perform case studies on reference sensitizers. The case studies aim to demonstrate real-life scenarios of performing safety assessment using only data from non-animal test methods (e.g., exposure data, skin bioavailability, in vitro test data for the first three AOP KEs and $\mathrm{T}$ cell assay data). The case studies would demonstrate the current limitations and might find that in some cases the available methods for the first three AOP KEs are not sufficient and additional information from $\mathrm{T}$ cell-based assays is required for safety assessment.

\section{Conclusions}

The development of AOP-driven integrated testing strategies, DAs and safety assessment approaches for skin sensitization still lack information on the fourth $\mathrm{KE}$, the antigen-specific $\mathrm{T}$ cell response. A number of $\mathrm{T}$ cell-based assays have shown encouraging test results in predicting the immunogenic potential of chemical substances, cosmetics ingredients and drug compounds (Guironnet et al., 2000; Delluc et al., 2011; Faulkner et al., 2012; Richter et al., 2013; Vocanson et al., 2014; Coulter et al., 2008; Gibson et al., 2015; Ahmed et al., 2016). Nevertheless, further optimization and refinement of these approaches are required to meet expectations regarding usability, predictive potential and reproducibility for regulatory safety assessment. A collaboration of experts on T cell-based assays from academia, industry and regulatory agencies will be important to overcome the remaining obstacles. Besides the further optimization of assay protocols, it is important to address the donor-to-donor variability and to identify the number of donors required to robustly detect $\mathrm{T}$ cell responses. Because $\mathrm{T}$ cell assays are relatively time-consuming and costly they may not be suitable as solely confirmatory assays. However, their application would add value if they provided additional information on potency. In view of regulatory acceptance, the advancement of $\mathrm{T}$ cell assays will also need to focus on standardization and transferability to allow ring-trial evaluation studies for predictive capacity and reproducibility assessment.

Though $\mathrm{T}$ cell-based assays for immunogenicity assessment are not that far away from a technical point of view, the use of corresponding $\mathrm{T}$ cell data for AOP-based safety assessment requires additional experience. To understand the value of $\mathrm{T}$ cell assays for safety assessment, case studies on real-life safety assessment scenarios may be highly informative. The case studies will demonstrate when information from $\mathrm{T}$ cell assays would be required and how the data could be integrated with other types of information in the safety assessment process. Although there are several alternative, non-animal test methods available covering innate immunity, how to use the test data from these assays to make decisions on the required safety factors still remains a challenge. Improved dose-response and sensitizer potency information for challenging reference substances may help to complete a skin sensitization safety assessment and define a threshold for a safe use concentration.

\section{References}

Ahmed, S. S., Wang. X. N., Fielding. M. et al. (2016). An in vitro human skin test for assessing sensitization potential. J Appl Toxicol 36, 669-684. doi:10.1002/jat.3197

Ashikaga, T., Yoshida, Y., Hirota, M. et al. (2006). Development of an in vitro skin sensitization test using human cell lines: The human cell line activation test (h-CLAT) I. Optimization of the h-CLAT protocol, Toxicol In Vitro 20, 767-773. doi:10.1016/j.tiv.2005.10.012

Basketter, D. A., Alépée, N., Ashikaga, T. et al. (2014). Categorization of chemicals according to their relative human skin sensitizing potency. Dermatitis 25, 11-21. doi:10.1097/ DER.0000000000000003

Burel, J. G., Qian, Y., Lindestam Arlehamn, C. et al. (2017). An integrated workflow to assess technical and biological variability of cell population frequencies in human peripheral blood by flow cytometry. J Immunol 198, 1748-1758. doi:10.4049/jimmunol.1601750

Castelli, F. A., Buhot, C., Sanson, A. et al. (2002). HLA-DP4, the most frequent HLA II molecule, defines a new supertype of peptide-binding specificity. J Immunol 169, 6928-6934. doi:10.4049/jimmunol.169.12.6928

Castelli, F. A., Szely, N., Olivain, A. et al. (2013). Hierarchy of CD4 T cell epitopes of the ANRS Lipo5 synthetic vac- 
cine relies on the frequencies of pre-existing peptide-specific T cells in healthy donors. J Immunol 190, 5757-5763. doi:10.4049/jimmunol.1300145

Corsini, E., Papale, A., Galbiati, V. and Roggen, E. L. (2014). Safety evaluation of cosmetic ingredients: In vitro opportunities for the identification of contact allergens. Cosmetics 1, 61-74. doi:10.3390/cosmetics1010061

Cortial, A., Nosbaum, A., Rozières, A. et al. (2015). Encapsulation of hydrophobic allergens into nanoparticles improves the in vitro immunological diagnosis of allergic contact dermatitis. Nanomedicine 11, 1029-1033. doi:10.1016/j. nano.2015.02.001

Cottrez, F., Boitel, E., Auriault, C. et al. (2015). Genes specifically modulated in sensitized skins allow the detection of sensitizers in a reconstructed human skin model. Development of the SENS-IS assay. Toxicol In Vitro 29, 787-802. doi:10.1016/j.tiv.2015.02.012

Coulter, E. M., Jenkinson, C., Wu, Y. et al. (2008). Activation of T-cells from allergic patients and volunteers by p-phenylenediamine and Bandrowski's base. J Invest Dermatol 128, 897-905. doi:10.1038/sj.jid.5701071

Delluc, S., Ravot, G. and Maillere, B. (2011). Quantitative analysis of the CD4 T-cell repertoire specific to therapeutic antibodies in healthy donors. FASEB J 25, 2040-2048. doi:10.1096/fj.10-173872

Dietz, L., Esser, P. R., Schmucker, S. S. et al. (2010). Tracking human contact allergens: From mass spectrometric identification of peptide-bound reactive small chemicals to chemical-specific naive human T-cell priming. Toxicol Sci 117, 336-347. doi:10.1093/toxsci/kfq209

Emter, R., Ellis, G. and Natsch, A. (2010). Performance of a novel keratinocyte-based reporter cell line to screen skin sensitisers in vitro. Toxicol Appl Pharmacol 245, 281-290. doi:10.1016/j.taap.2010.03.009

Emtestam, L., Zetterquist, H. and Olerup, O. (1993). HLADR, -DQ and -DP alleles in nickel, chromium, and/or cobalt-sensitive individuals: Genomic analysis based on restriction fragment length polymorphisms. J Invest Dermatol 100, 271-274. doi:10.1111/1523-1747.ep12469732

Esser, P. R., Kimber, I. and Martin, S. F. (2014). Correlation of contact sensitizer potency with T cell frequency and TCR repertoire diversity. EXS 104, 101-114. doi:10.1007/978-30348-0726-5_8

EC (2009). Regulation No. 1223/2009 of the European Parliament and of the Council of 30 November 2009 on cosmetic products. http://eur-lex.europa.eu/legal-content/EN/ ALL/?uri=CELEX\%3A32009R 1223

Ezendam, J., Braakhuis, H. M. and Vandebriel, R. J. (2016). State of the art in non-animal approaches for skin sensitization testing: From individual test methods towards testing strategies. Arch Toxicol 90, 2861-2883. doi:10.1007/ s00204-016-1842-4

Faulkner, L., Martinsson, K., Santoyo-Castelazo, A. et al. (2012). The development of in vitro culture methods to characterize primary T-cell responses to drugs. Toxicol Sci
127, 150-158. doi:10.1093/toxsci/kfs080

Friedmann, P. S., Moss, C., Shuster, S. and Simpson J. M. (1983). Quantitative relationships between sensitizing dose of DNCB and reactivity in normal subjects. Clin Exp Immunol 53, 709-715.

Gerberick, G. F., Vassallo, J. D., Bailey, R. E. et al. (2004). Development of a peptide reactivity assay for screening contact allergens. Toxicol Sci 81, 332-343. doi:10.1093/ toxsci/kfh213

Gerberick, G. F., Ryan, C. A., Dearman, R. J. and Kimber, I. (2007). Local lymph node assay (LLNA) for detection of sensitization capacity of chemicals. Methods 41, 54-60. doi:10.1016/j.ymeth.2006.07.006

Gerberick, G. F., Troutman, J. A., Foertsch, L. M. et al. (2009). Investigation of peptide reactivity of prohapten skin sensitisers using a peroxidase-peroxide oxidation system. Toxicol Sci 112, 164-174. doi:10.1093/toxsci/kfp192

Gibbs, S., Corsini, E., Spiekstra, S. W. et al. (2013). An epidermal equivalent assay for identification and ranking potency of contact sensitizers. Toxicol Appl Pharmacol 272, 529-541. doi:10.1016/j.taap.2013.07.003

Gibson, A., Kim, S. H., Faulkner, L. et al. (2015). In vitro priming of naïve T-cells with p-phenylenediamine and Bandrowski's base. Chem Res Toxicol 28, 2069-2077. doi:10.1021/acs.chemrestox.5b00294

Giese, C. and Marx, U. (2014). Human immunity in vitro Solving immunogenicity and more. Adv Drug Deliv Rev 69-70, 103-122. doi:10.1016/j.addr.2013.12.011

Guironnet, G., Dalbiez-Gauthier, C., Rousset, F. et al. (2000). In vitro human $\mathrm{T}$ cell sensitization to haptens by monocyte-derived dendritic cells. Toxicol In Vitro 14, 517-522. doi:10.1016/S0887-2333(00)00043-6

Hoffmann, S., Kleinstreuer, N., Alépée, N. et al. (2018). Non-animal methods to predict skin sensitization (I): The Cosmetics Europe database. Crit Rev Toxicol, Feb 23. Epub ahead of print. doi:10.1080/10408444.2018.1429385

Hooyberghs, J., Schoeters, E., Lambrechts, N. et al. (2008). A cell-based in vitro alternative to identify skin sensitizers by gene expression. Toxicol Appl Pharmacol 231, 103-111. doi:10.1016/j.taap.2008.03.014

Hou, X. L., Wang, L., Ding, Y. L. et al. (2016). Current status and recent advances of next generation sequencing techniques in immunological repertoire. Genes Immun 17, 153164. doi:10.1038/gene.2016.9

Jaworska, J. S. (2016). Integrated testing strategies for skin sensitization hazard and potency assessment - State of the art and challenges. Cosmetics 3, 16. doi:10.3390/cosmetics3020016

Johansson, H., Albrekt, A. S., Borrebaeck, C. A. and Lindstedt, M. (2013). The GARD assay for assessment of chemical skin sensitisers. Toxicol In Vitro 37, 1163-1169. doi:10.1016/j.tiv.2012.05.019

Jowsey, I. R., Basketter, D. A., Westmoreland, C. and Kimber, I. (2006). A future approach to measuring relative skin sensitising potency: A proposal. J Appl Toxicol 26, 341-350. doi:10.1002/jat.1146 
Kimber, I., Dearman, R. J., Basketter, D. A. et al. (2002a). The local lymph node assay: Past, present and future. Contact Dermatitis 47, 315-328. doi:10.1034/j.16000536.2002.470601.x

Kimber, I., Basketter, D. A., Gerberick, G. F. and Dearman, R. J. (2002b). Allergic contact dermatitis. Int Immunopharmacol 2, 201-211. doi:10.1016/S1567-5769(01)00173-4

Kimber, I., Maxwell, G., Gilmour, N. et al. (2012). Allergic contact dermatitis: A commentary on the relationship between T lymphocytes and skin sensitising potency. Toxicology 291, 18-24. doi:10.1016/j.tox.2011.11.007

Kimura, Y., Fujimura, C., Ito, Y. et al. (2015). Optimization of the IL-8 Luc assay as an in vitro test for skin sensitization. Toxicol In Vitro 29, 1816-1830. doi:10.1016/j. tiv.2015.07.006

Kleinstreuer, N., Hoffmann, S., Alépée, N. et al. (2018). Non-animal methods to predict skin sensitization (II): An assessment of defined approaches. Crit Rev Toxicol, Feb 23. Epub ahead of print. doi:10.1080/10408444.2018.142 9386.

MacKay, C., Davies, M., Summerfield, V. and Maxwell, G. (2013). From pathways to people: Applying the adverse outcome pathway (AOP) for skin sensitization to risk assessment. ALTEX 30, 473-486. doi:10.14573/altex.2013.4.473

Maxwell, G., MacKay, C., Cubberley, R. et al. (2014). Applying the skin sensitisation adverse outcome pathway (AOP) to quantitative risk assessment. Toxicol In Vitro 28, 8-12. doi:10.1016/j.tiv.2013.10.013

McKim, J. M., Jr., Keller, D. J., $3^{\text {rd }}$ and Gorski, J. R. (2012). An in vitro method for detecting chemical sensitisation using human reconstructed skin models and its applicability to cosmetic, pharmaceutical, and medical device safety testing. Cutan Ocul Toxicol 31, 292-305. doi:10.3109/1556 9527.2012.667031

Mehling, A., Eriksson, T., Eltze, T. et al. (2012). Non-animal test methods for predicting skin sensitization potentials. Arch Toxicol 86, 1273-1295. doi:10.1007/s00204-0120867-6

Natsch, A., Bauch, C., Foertsch, L. et al. (2011). The intraand inter-laboratory reproducibility and predictivity of the KeratinoSens assay to predict skin sensitisers in vitro: Results of a ring-study in five laboratories. Toxicol In Vitro 25, 733-744. doi:10.1016/j.tiv.2010.12.014

OECD (2012). OECD Series on Testing and Assessment No. 168. The Adverse Outcome Pathway for Skin Sensitisation Initiated by Covalent Binding to Proteins. Part 1: Scientific Evidence. Paris, OECD Publishing. http://bit.ly/1EEvQoq

OECD (2015a). Test No. 442C: In Chemico Skin Sensitisation. Direct Peptide Reactivity Assay (DPRA), OECD Guidelines for the Testing of Chemicals, Section 4 ed. OECD, Paris. doi:10.1787/20745788

OECD (2015b). Test No. 442D: In Vitro Skin Sensitisation. In Vitro Skin Sensitisation: ARE-Nrf2 Luciferase Test Method, OECD Guidelines for the Testing of Chemicals, Section 4 ed. OECD, Paris. doi:10.1787/20745788
OECD (2016). Guidance Document on the Reporting of Defined Approaches and Individual Information Sources to be Used Within Integrated Approaches to Testing and Assessment (IATA) for Skin Sensitisation. Series on Testing \& Assessment, No. 256, Paris, OECD Publishing. http://bit. $1 \mathrm{y} / 2 \mathrm{wYhzVN}$

OECD (2017). In Vitro Skin Sensitisation assays addressing the Key OECD. Test No. 442E: Event on activation of dendritic cells on the Adverse Outcome Pathway for Skin Sensitisation. OECD, Paris. doi:10.1787/9789264264359-en

Piroird, C., Ovigne, J. M., Rousset, F. et al. (2015). The myeloid U937 skin sensitization test (U-SENS) addresses the activation of dendritic cell event in the adverse outcome pathway for skin sensitization, Toxicol In Vitro 29, 901-916. doi:10.1016/j.tiv.2015.03.009

Ramirez, T., Mehling, A., Kolle, S. N. et al. (2014). LuSens: A keratinocyte based ARE reporter gene assay for use in integrated testing strategies for skin sensitization hazard identification. Toxicol In Vitro 28, 1482-1497. doi:10.1016/j. tiv.2014.08.002

Reisinger, K., Hoffmann, S., Alepee, N. et al. (2015). Systematic evaluation of non-animal test methods for skin sensitisation safety assessment. Toxicol In Vitro 29, 259-270. doi:10.1016/j.tiv.2014.10.018

Reuter, H., Spieker, J., Gerlach, S. et al. (2011). In vitro detection of contact allergens: Development of an optimized protocol using human peripheral blood monocyte-derived dendritic cells. Toxicol In Vitro 25, 315-323. doi:10.1016/j. tiv.2010.09.016

Richter, A., Schmucker, S. S., Esser, P. R. et al. (2013). Human $\mathrm{T}$ cell priming assay (hTCPA) for the identification of contact allergens based on naive T cells and DC - IFN- $\gamma$ and TNF- $\alpha$ readout. Toxicol In Vitro 27, 1180-1185. doi:10.1016/j.tiv.2012.08.007

Rombach-Riegraf, V., Karle, A. C., Wolf, B. et al. (2014). Aggregation of human recombinant monoclonal antibodies influences the capacity of dendritic cells to stimulate adaptive T-cell responses in vitro. PLoS One 9, e86322. doi:10.1371/ journal.pone.0086322

Scornet, N., Delarue-Cochin, S., Azoury, M. E. et al. (2016). Bioinspired design and oriented synthesis of immunogenic site-specifically penicilloylated peptides. Bioconjug Chem 27, 2629-2645. doi:10.1021/acs.bioconjchem.6b00393

Sullivan, A., Wang, E., Farrell, J. et al. (2017). $\beta$-Lactam hypersensitivity involves expansion of circulating and skin-resident TH22 cells. J Allergy Clin Immunol 141, 235249. doi:10.1016/j.jaci.2017.01.020

Texier, C., Pouvelle, S., Busson, M. et al. (2000). HLA-DR restricted peptide candidates for bee venom immunotherapy. $J$ Immunol 164, 3177-3184. doi:10.4049/jimmunol.164.6.3177

Texier, C., Pouvelle-Moratille, S., Busson, M. et al. (2001). Complementarity and redundancy of the binding specificity of HLA-DRB1, -DRB3, -DRB4 and -DRB5 molecules. Eur J Immunol 31, 1837-1846. doi:10.1002/15214141(200106)31:6<1837::AID-IMMU1837>3.0.CO;2-H 
Thierse, H. J., Budde, P., Dietz, L. et al. (2011). Proteomic identification of allergen-regulated proteins and allergen-protein interaction networks in assisting biomarker and assay development. In E. L. Roggen, H. U. Weltzien, H. Helma (eds.), Progress Towards Novel Testing Strategies for In Vitro Assessment of Allergens (145-166). Kerala, India: Transworld Research Network.

Vocanson, M,. Achachi, A., Mutez, V. et al. (2014). Human $\mathrm{T}$ cell priming assay: Depletion of peripheral blood lymphocytes in CD25+ cells improves the in vitro detection of weak allergen-specific T cells. EXS 104, 89-100. doi:10.1007/978-3-0348-0726-5_7

Vukmanović, S. and Sadrieh, N. (2017). Skin sensitizers in cosmetics and beyond: Potential multiple mechanisms of action and importance of T-cell assays for in vitro screening. Crit Rev Toxicol 22, 1-18. doi:10.1080/10408444.2017 .1288025

Wang, P., Sidney, J., Dow, C. et al. (2008). A systematic assessment of MHC class II peptide binding predictions and evaluation of a consensus approach. PLoS Comput Biol 4, e1000048. doi:10.1371/journal.pcbi.1000048
White, K. D., Chung, W. H., Hung, S. I. et al. (2015). Evolving models of the immunopathogenesis of T cell-mediated drug allergy: The role of host, pathogens, and drug response. $J$ Allergy Clin Immunol 136, 219-234. doi:10.1016/j. jaci.2015.05.050

Wong, C. L., Ghassabian, S., Smith, M. T. and Lam, A. L. (2015). In vitro methods for hazard assessment of industrial chemicals - Opportunities and challenges. Front Pharmacol 6, 94. doi:10.3389/fphar.2015.00094

\section{Conflict of interest}

Cosmetics Europe has financially supported the development and optimization of the human T Cell Priming Assay (hTCPA).

\section{Correspondence to}

Martina Klaric, $\mathrm{PhD}$

Cosmetics Europe

Avenue Herrmann Debroux 40

1160 Brussels, Belgium

Phone: +322227 6631

e-mail: mklaric@cosmeticseurope.eu 